\title{
Anonymous Secure Communication in Wireless Mobile Ad-hoc Networks
}

\author{
Sk. Md. Mizanur Rahman ${ }^{\ddagger 1}$, Atsuo Inomata ${ }^{\S}$, Takeshi Okamoto ${ }^{\ddagger 2}, \quad$ Masahiro Mambo ${ }^{\star 3}$ and \\ Eiji Okamoto $\$ 4$ \\ $\$$ Graduate School of Systems and Information Engineering, University of Tsukuba, Japan \\ $\S$ Japan Science and Technology agency \\ 1 e-mail: mizanur@cipher.risk.tsukuba.ac.jp \\ $\S$ e-mail: inomata@ristex.jst.go.jp \\ ${ }^{2}$ e-mail: ken@risk.tsukuba.ac.jp \\ 3 e-mail: mambo@cs.tsukuba.ac.jp \\ ${ }^{4}$ e-mail: okamoto@,risk.tsukuba.ac.jp
}

\begin{abstract}
The main characteristic of a mobile ad-hoc network is its infrastructure-less, highly dynamic topology, which is subject to malicious traffic analysis. Malicious intermediate nodes in wireless mobile ad-hoc networks are a threat concerning security as well as anonymity of exchanged information. To protect anonymity and achieve security of nodes in mobile ad-hoc networks, an anonymous on-demand routing protocol, termed RIOMO, is proposed. For this purpose, pseudo IDs of the nodes are generated considering Pairing-based Cryptography. Nodes can generate their own pseudo IDs independently. As a result RIOMO reduces pseudo IDs maintenance costs. Only trust-worthy nodes are allowed to take part in routing to discover a route. To ensure trustiness each node has to make authentication to its neighbors through an anonymous authentication process. Thus RIOMO safely communicates between nodes without disclosing node identities; it also provides different desirable anonymous properties such as identity privacy, location privacy, route anonymity, and robustness against several attacks.
\end{abstract}

Keywords: Ad-hoc network, Anonymity, Routing, Pairing-Based Cryptography, Security

\section{Introduction}

Conventional wireless mobile communications are normally supported by a fixed wire/wireless infrastructure. A mobile device would use a single-hop wireless radio communication to access a fixed base-station that connect it to the wire/wireless infrastructure. In contrast, ad-hoc networks do not use any fixed infrastructure. The nodes in a mobile ad-hoc network intercommunicate via single-hop and multi-hop paths in a peer-to-peer fashion. Intermediate nodes between a pair of communicating nodes act as a routers [1]. Thus the nodes operate both as hosts and routers. The nodes in the ad-hoc network could be potentially mobile, and so the creation of routing paths is affected by the addition and deletion of nodes. The topology of the network may change randomly, rapidly, and unexpectedly [1].

Mobile ad hoc networks, MANETs, are finding ever-increasing applications in both military and civilian systems owing to their self-configuration and self-maintenance capabilities. Collaborative computing and communications in smaller areas (building organizations, conference, etc.) can set up using as-hoc networking technologies [1]. Communications in battlefields and disaster recovery are other examples of application environments. Many of these applications, such as military battlefield operations, homelandsecurity scenarios, law enforcement, and rescue missions are security sensitive. As a result, security in MANETs has recently been drawing much attention [2].

Traffic analysis is one of the most subtle and unsolved security attacks against MANETs. By definition, it is an attack such that an adversary observes network traffic and infers sensitive information of the applications and/or the underlying system [3]. Sensitive information includes the identities of communicating parties, network traffic patterns [2], and their changes. The leakage of such information is often devastating in security-sensitive scenarios. For example, an unexpected change of the traffic pattern 
in a military network may indicate a forthcoming action, a chain of commands, or a state change of network alertness [4]. It may also reveal the locations of command centers or mobile VIP nodes, which will enable the adversaries to launch pinpoint attacks on them. In contrast to active attacks, which usually involve the launch of denial of service or other more "visible" and aggressive attacks on the target network, traffic analysis is a kind of passive attack, which is "invisible" and difficult to detect. It is therefore important to design countermeasures against such malicious traffic analysis.

The shared wireless medium of MANETs introduces opportunities for passive eavesdropping on data communications. Adversaries can easily overhear all messages "flying in the air" without physically compromising nodes. Several methods for withstanding eavesdropping and other kinds of traffic analysis have been investigated $[5,6]$. There is other approach is to perform end-to-end encryption and/or link encryption on data traffic. However, this only prevents adversaries from accessing traffic contents. Adversaries can still carry out traffic analysis based on the bare network-layer and/or MAC addresses, both of which are unprotected and unencrypted in common ad-hoc routing protocols such as Ad hoc OnDemand Distance Vector (AODV) [7], the Dynamic Source Routing Protocol for Mobile Ad Hoc Networks (DSR) [8], and the de facto MAC protocol IEEE 802.11. Unfortunately these protocols were not designed to be secure and do not defend against malicious attacks. AODV and DSR, two protocols under consideration for standardization by the IETF MANET Working Group, are both vulnerable to a number of attacks including impersonation, modification, and fabrication [9].

In this paper another property of security, namely anonymity and/or privacy is discussed in terms of adhoc network communication. Anonymity is one of the most important factors for securing ad-hoc network communications, where the intruders do not know about the communication IDs. It ensures that a user may use a resource or service without disclosing the user's identity. The requirements for anonymity provide protection of the user identity. Anonymity requires that other users or subjects are unable to determine the identity of a user bound to a subject or operation [10]. Anonymity is the stronger; the less is known about the linking to a subject. As a result, adversaries fail to make correlation between the eavesdropped traffic information and the actual network traffic patterns. Thus traffic analysis attack can be efficiently defeated. The strength of anonymity decreases with increasing knowledge of the pseudonym linking. To keep the strength of anonymity strong, it should be mind in designing anonymous protocol that intruders can not increase their knowledge about pseudonym linking. With this view; to achieve strong communication anonymity and security, an anonymous on-demand routing protocol, called RIOMO, is proposed. In RIOMO, every node can generate its own pseudo IDs dynamically based-on pairing-based cryptography and random numbers; also nodes can generate these pseudo IDs independently, without making communication with the system administrator. Thus pseudo IDs maintenance cost is reduced compared to the previous proposed method namely MASK by Zhang et al., [11]. These pseudo IDs of the nodes are used for communication. On the other hand the intruders can not define pseudonym linking with the node pseudo IDs, so traffic analysis attack is prevented. A route is discovered without disclosing the nodes IDs for successful communication.

The rest of this paper is organized as follows. In section 2, preliminaries are described. In section 3, RIOMO architecture and design are given. In section 4 RIOMO protocol is described. In section 5 anonymity achievements and security analysis are given. Finally, section 6 describes conclusions and future works.

\subsection{Related Work and Our Contributions}

The proposed protocol RIOMO is exclusively based on the pairing-based cryptographic properties. There is also another approach of anonymous communication based on pairing-based cryptography proposed by Zhang et al., [11], called MASK. In MASK, system administrator generates a large set of pseudo IDs for every node. So, in MASK, every node has a fixed pseudo ID set and it should large enough set, otherwise there is a chance of finding pseudonym linking by the intruders as a result anonymity decrease and it fails to full-fill its target. If the pseudo ID set for a node is small then anonymity property lose of the MASK protocol, because every node has to repeat its pseudo IDs after finishing one round of all its pseudo IDs. Thus pseudo IDs work as real IDs and intruders able to identify each node. So, to keep strong anonymity in MASK, every node should have to manage an extremely large enough number of pseudo IDs set provided by the system administrator, which is costly for ad-hoc network communication in terms of extra task for nodes, IDs maintenance. In this paper we explicitly show that; by using only one pseudo ID taking from 
system administrator, nodes can generate their own pseudo IDs independently and dynamically. It is the first approach to achieve anonymity by using only one pseudo ID taking from the system administrator in ad-hoc network. With pairing based IBE properties and random number nodes can generate their own pseudo IDs dynamically, which also provide strong security properties.

There are some other proposals $[12,13,14,15]$ taking care of privacy. In [12], a secure dynamic distributed routing algorithm (denoted as SDDR in this paper) for ad hoc wireless networks is proposed based on the onion routing protocol [13]. The anonymity-related properties achieved in this algorithm include weak location privacy and route anonymity. However, it ignores one important part of privacy in mobile ad-hoc networks, namely identity anonymity, and it cannot provide strong location privacy.

In [14], Kong et al. design an Anonymous On-Demand Routing (ANODR) based on topology. Similar to Hordes [15], ANODR also applies multicast/broadcast to improve recipient anonymity. ANODR is an ondemand protocol, and is based on trapdoor information in the broadcast. These features are not discussed in regards to Hordes' [15] multicast mechanism.

Compared to [12], ANODR gives a more comprehensive analysis of the anonymity and security properties achieved, and provide detailed simulation results. In addition, ANODR is more efficient than SDDR at the data-transmission stage. However, similar to SDDR in [12], ANODR does not provide identity anonymity and strong location privacy. RIOMO and other two protocols are described in Table 1 and in Table 2 with respect to the Anonymity-related properties and security-related properties respectively. Detailed discussions of these properties are given in Section 5.

Table 1. Comparison of Anonymity-related properties

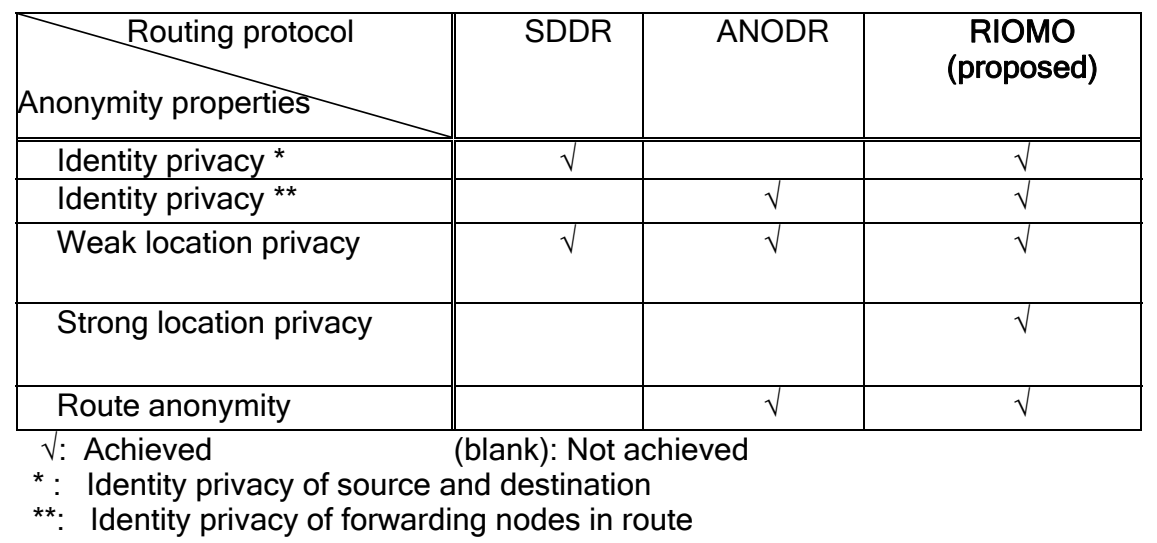

Table 2. Comparison of security-related properties

\begin{tabular}{|c||c|c|c|}
\hline Routing protocol & SDDR & ANODR & $\begin{array}{c}\text { RIOMO } \\
\text { (proposed) }\end{array}$ \\
\hline \hline Dos attacks & & & $\sqrt{ }$ \\
\hline Wormhole attacks & $\sqrt{ }$ & $\sqrt{ }$ & $\sqrt{ }$ \\
\hline Rushing attacks & $\sqrt{ }$ & $\sqrt{ }$ & $\sqrt{ }$ \\
\hline
\end{tabular}

$\sqrt{ }$ : Protected (blank): Not protected

\section{Preliminaries}

\subsection{Characteristics of Wireless Communication System.}

One of the major challenges in ad hoc networks security is that ad hoc networks typically lack of a fixed infrastructure both in form of physical infrastructure such as routers, servers and stable communication 
links and in the form of an organizational or administrative infrastructure [16]. Another difficulty lies in the highly dynamic nature of ad hoc networks since new nodes can join and leave the network at any time. The major problem in providing security services in such infrastructure-less networks lies on how to manage the cryptographic keys that are needed. When designing protocols for ad hoc networks, whether routing protocols or security protocols, it is important to consider the characteristics of the network and realize that there are many "flavours" of ad hoc networks. Ad hoc wireless networks generally have the following characteristics [17]:

Dynamic network topology: The network nodes are mobile and thus the topology of the network may change frequently. Nodes may move around within the network, the network can be partitioned into multiple smaller networks or be merged with other networks.

Limited bandwidth: The use of wireless communication typically implies a lower bandwidth than that of traditional networks. This may limit the number and size of messages sent during protocol execution.

Energy constrained nodes: Nodes in ad hoc networks will most often rely on batteries as their power source. The use of computationally complex algorithms may not be possible. This also exposes the nodes to a new type of denial of service attack, the sleep deprivation torture attack [17] that aims at depleting the nodes energy source.

Limited physical security: The use of wireless communication and the exposure of the network nodes increase the possibility of attacks against the network. Due to the mobility of the nodes the risk of them being physically compromised by theft, loss or other means will probably be greater than that for traditional network nodes. In many cases the nodes of ad hoc network may also have limited CPU performance and memory, e.g. low-end devices such as PDA's, cellular phones and embedded devices. As a result certain algorithms that are computationally or memory expensive might not be applicable.

\subsection{Bilinear maps}

Let $G_{1}$ an additive group and $G_{2}$ be a multiplicative group of the same prime order $q$. Let $P$ be an arbitrary generator of $G_{I}$. (aP denotes $P$ added to itself a times). Assume that discrete logarithm (DL) problem is hard in both $G_{I}$ and $G_{2}$. We can think $G_{1}$ as a group of points on an elliptic curve over $F_{q}$, and $G_{2}$ as a subgroup of the multiplicative group of a finite field $F_{q^{k}}$ for some $k \in Z_{q}^{*}$. A mapping $\tilde{e}: G_{l} \times G_{l} \rightarrow G_{2}$, satisfying the following properties is called a cryptographic bilinear map.

- Bilinearity: $\tilde{e}(a P, b Q)=\tilde{e}(P, Q)^{a b}$ for all $P, Q \in G_{l}$ and $a, b \in Z_{q}^{*}$. This can be restated in the following way. For $P, Q, R \in G_{l}, \tilde{e}(P+Q, R)=\tilde{e}(P, R) \tilde{e}(Q, R)$ and $\tilde{e}(P, Q+R)=\tilde{e}(P, Q) \tilde{e}(P, R)$.

- Non-degeneracy: If $P$ is a generator of $G_{1}$, then $\tilde{e}(P, P)$ is a generator of $G_{2}$. In other words, $\tilde{e}(P, P) \neq 1$.

- Computable: A mapping is efficiently computable if $\tilde{e}(P, P)$ can be computed in polynomial-time for all $P, Q \in G_{1}$.

Modified Weil Pairing [18] and Tate Pairing [19, 20] are examples of cryptographic bilinear maps.

\subsection{Diffie-Hellman Problems}

With the group $G_{I}$ described in section 2.2, we can define the following hard cryptographic problem applicable to our proposed scheme.

- Discrete Logarithm (DL) Problem: Given $P, Q \in G_{l}$, find an integer $n$ such that $P=n Q$ whenever such integer exists.

- Computational Diffie-Hellman (CDH) Problem: Given a triple $(P, a P, b P) \in G_{l}$ for $a, b \in Z_{q}^{*}$, find the element $a b P$.

- Decision Diffie-Hellman (DDH) problem: Given a quadruple $(P, a P, b P, c P) \in G_{l}$ for $a, b, c \in Z_{q}^{*}$, decide whether $c=a b \bmod q$ or not.

- Gap Diffie-Hellman (GDH) Problem: A class of problems where the CDH problem is hard but DDH problem is easy.

- Bilinear Diffie-Hellman (BDH) Problem: Given a quadruple $(P, a P, b P, c P) \in G_{l}$ for some $a, b, c \in$ $Z_{q}{ }^{*}$, compute $\tilde{e}(P, P)^{a b c}$. 
Groups where the $\mathrm{CDH}$ problem is hard but DDH problem is easy are called GAP Diffie-Hellman $(\mathrm{GDH})$ groups. Details about GDH groups can be found in [21, 22, 23].

\section{RIOMO Architecture and Design}

In RIOMO, system administrator does not take part in routing rather it has the following tasks during the boot strap of the network.

- Determines two groups $G_{1}, G_{2}$, of the same prime order $q$. We view $G_{I}$ as an additive group and $G_{2}$ as a multiplicative group as discussed in section 2.2.

- Determines bilinear map g: $G_{1} \times G_{1} \rightarrow G_{2}$, collision resistant cryptographic hash functions $\mathrm{H}_{1}$ and $\mathrm{H}_{2}$, where $\mathrm{H}_{1}:\{0,1\}^{*} \rightarrow G_{l}$ mapping from arbitrary-length strings to points in $G_{I}$ and $\mathrm{H}_{2}$ : $\{0,1\}^{*} \rightarrow\{0,1\}^{\mu}$ mapping from arbitrary-length strings to $\mu$-bit fixed length output.

- Generates system's secret $\boldsymbol{\omega} \in \mathrm{Z}_{q}^{*}$, where $\mathrm{Z}_{q}{ }^{*}=\{\mathrm{y} \mid 1 \leq \mathrm{y} \leq q-1\}$. Any one in the network does not know $\boldsymbol{\omega}$ except system administrator. System administrator also uses this secret to generate the secret point of the non-adversary nodes.

Thus the system parameters $<\mathrm{G}_{1}, \mathrm{G}_{2}, \mathrm{~g}, \mathrm{H}_{1}, \mathrm{H}_{2}>$ are known to the non-adversary nodes. System administrator also provides the following parameters for nodes, regarding their IDs and secret points.

- Provides each node, a secret point $\mathrm{SP}_{\mathrm{R}}$, with respect to the node's real ID ID $\mathrm{ID}_{\mathrm{R}}$, which is defined as $\mathrm{SP}_{\mathrm{R}}=\boldsymbol{\omega} \mathrm{H}_{1}\left(\mathrm{ID}_{\mathrm{R}}\right)$. The Source and the destination use their corresponding secret point in the route discovery phase to authenticate each other. For a given set of $<\mathrm{ID}_{\mathrm{R}}, \mathrm{SP}_{\mathrm{R}}>$ no one can determine the system secret $\boldsymbol{\omega}$ as we discussed in section 2.3.

- Provides each node a different pseudo ID $\operatorname{IDP}_{\mathrm{i}}$, and their corresponding secret point $\mathrm{SPP}_{\mathrm{i}}$, which is defined as $\mathrm{SPP}_{\mathrm{i}}=\boldsymbol{\omega}_{\mathrm{H}_{1}}\left(\mathrm{IDP}_{\mathrm{i}}\right)$; if $\mathrm{i} \neq \mathrm{j}$ then $\mathrm{IDP}_{\mathrm{i}} \neq \mathrm{IDP}_{\mathrm{j}}$ as well as $\mathrm{SPP}_{\mathrm{i}} \neq \mathrm{SPP}_{\mathrm{j}}$. For a given set of $<\mathrm{IDP}_{\mathrm{i}}, \mathrm{SPP}_{\mathrm{i}}>$ also no one can determine the system secret $\boldsymbol{\omega}$.

With the above information any node can generate its own pseudo IDs and the corresponding secret points randomly in every session in communication. Let's check for node $\mathrm{k}$; $\mathrm{k}$ has received its pseudo ID $\mathrm{IDP}_{\mathrm{k}}$ and the corresponding secret point $\mathrm{SPP}_{\mathrm{k}}=\boldsymbol{\omega} \mathrm{H}_{1}\left(\mathrm{IDP}_{\mathrm{k}}\right)$ from the system administrator. So, $\mathrm{k}$ can generate its own pseudo ID $\mathrm{ID}_{\mathrm{Pk}_{\mathrm{k}}}=\mathrm{R}_{\mathrm{k}} \mathrm{H}_{1}\left(\mathrm{IDP}_{\mathrm{k}}\right)$, and the corresponding secret point $\mathrm{SP}_{\mathrm{Pk}}=\mathrm{R}_{\mathrm{k}} \mathrm{SPP}_{\mathrm{k}}=\mathrm{R}_{\mathrm{k}} \boldsymbol{\omega} \mathrm{H}_{1}\left(\mathrm{IDP}_{\mathrm{k}}\right)=\boldsymbol{\omega} \mathrm{R}_{\mathrm{k}} \mathrm{H}_{1}\left(\mathrm{IDP}_{\mathrm{k}}\right)=\boldsymbol{\omega} \mathrm{ID}_{\mathrm{Pk}}$ where $\mathrm{R}_{\mathrm{k}}$ is a random generated by $\mathrm{k}$; this equation also holds the previous cited property in section 2 that no one can determine the system secret $\boldsymbol{\omega}$ for a given set of pseudo ID and the corresponding secret point, $<\mathrm{ID}_{\mathrm{Pk}}, \mathrm{SP}_{\mathrm{Pk}}>$. Thus a node can generate its own pseudo IDs and corresponding secret points as its need.

\section{RIOMO Protocol}

\subsection{Anonymous Neighbor Authentication}

When a node wants to join in the network or moves to a new place, it has to authenticate within its neighbor nodes. Say, Alice has received her pseudo ID $\mathrm{IDP}_{\mathrm{A}}$, and the corresponding secret point $\mathrm{SPP}_{\mathrm{A}}=\boldsymbol{\omega}_{\mathrm{H}}\left(\mathrm{IDP}_{\mathrm{A}}\right)$, i.e., $<\mathrm{IDP}_{\mathrm{A}}, \mathrm{SPP}_{\mathrm{A}}>$ from the system administrator. She can join in the network by authenticating within her neighbor nodes or if she moves another place in the network different from her current place, she also needs to authenticate her within her neighbor. To avoid an attack, if Alice wants to change her pseudo ID different from her current pseudo ID without moving her place, she also needs to authenticate her current pseudo ID within her neighbor. For this purpose she generates pseudo ID $\mathrm{ID}_{\mathrm{PA}}=\mathrm{R}_{\mathrm{A}} \mathrm{H}_{1}\left(\mathrm{IDP}_{\mathrm{A}}\right)$, corresponding secret point $\mathrm{SP}_{\mathrm{PA}}=\mathrm{R}_{\mathrm{A}} \mathrm{SPP}_{\mathrm{A}}=\mathrm{R}_{\mathrm{A}} \boldsymbol{\omega} \mathrm{H}_{1}\left(\mathrm{IDP}_{\mathrm{A}}\right)=\boldsymbol{\omega} \mathrm{R}_{\mathrm{A}} \mathrm{H}_{1}\left(\operatorname{IDP}_{\mathrm{A}}\right)=\boldsymbol{\omega} \mathrm{ID}_{\mathrm{PA}}$, where $R_{A}$ is a random generated by Alice; she also generates a random $R_{R A}$ which is used to generate verification codes $\operatorname{Ver}_{0}{ }^{*}$ and $V_{e}$. Alice broadcasts her pseudo ID $\mathrm{ID}_{\mathrm{PA}}$, and a random $\mathrm{R}_{\mathrm{RA}}$ within her neighbor region. One of her neighbor, let's say Bob, makes a response with his pseudo ID ID $\mathrm{PB}_{\mathrm{PB}}$, generated random $\mathrm{R}_{\mathrm{RB}}$ and verification code $\mathrm{Ver}_{0}$ as shown in fig.1. If Alice is a valid node then $\operatorname{Ver}_{0}{ }^{*}=\mathrm{Ver}_{0}$, and $\operatorname{Ver} 1^{*}=\operatorname{Ver}_{1}$ thus she can be a member and she is identified as $\mathrm{ID}_{\mathrm{PA}}$, within her neighbor. Thus Alice and Bob use their session key $\mathrm{K}_{\mathrm{AB}}=\mathrm{K}_{\mathrm{BA}}$ corresponding their pseudo IDs $\mathrm{ID}_{\mathrm{PA}}$, $\mathrm{ID}_{\mathrm{PB}}$, respectively. No one within Alice's neighbor can recognize her as Alice because she is using her pseudo ID and she is changing her 
pseudo ID time to time. Thus the nodes can hide their IDs in the network and always seem new to each other. Any adversary node can not be a member within its neighbor, because it has to pass the verification code "? (Ver1*= Ver1)" which is not possible to generate without the knowledge of the system secret. Similar way all nodes in the network can authenticate anonymously within their neighbors and generate their corresponding session key. Thus nodes in the network maintain their neighbor table with their pseudo IDs and corresponding session key.

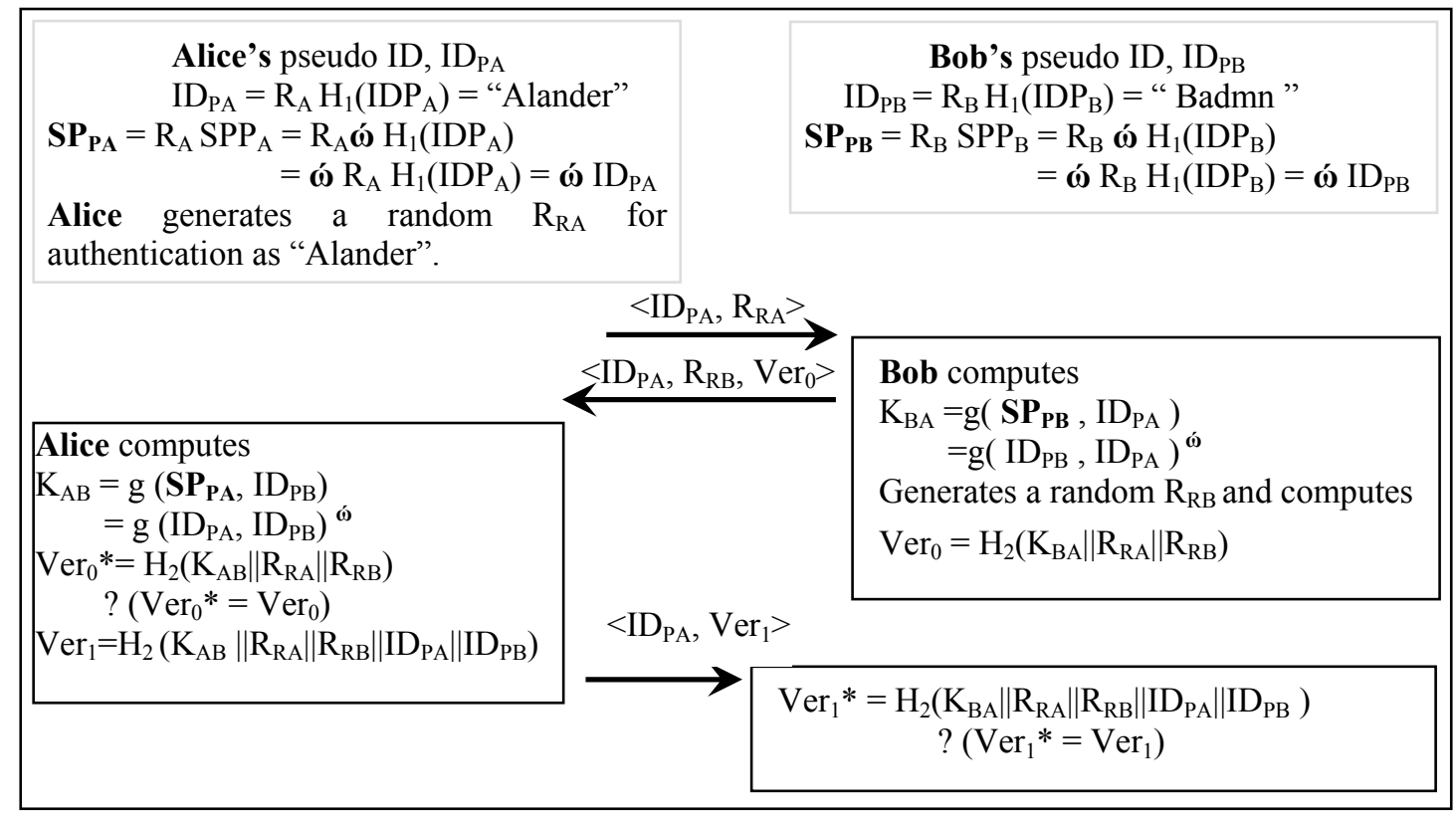

Fig. 1. Anonymous neighbor authentication process for two neighbor nodes "Alice" and "Bob"

\subsection{Control Packets}

RIMIO uses route request packet $R R Q$, and route reply packet $R R P$, to find a route in the network. To discover a route and to receive a response it uses $R R Q$ and $R R P$ respectively.

RRQ

\begin{tabular}{|l|l|l|l|}
$\mathrm{ID}_{\mathrm{PSE}}$ & $R R Q S e q N O$ & $\mathrm{ID}_{\mathrm{S}}$ & $\mathrm{ID}_{\mathrm{D}}$ \\
\hline
\end{tabular}

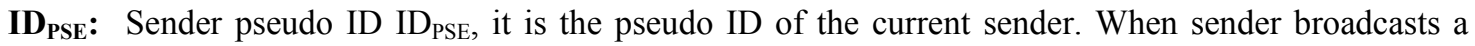
$R R Q$ packet it puts its own pseudo ID in this field. Thus $\mathrm{ID}_{\mathrm{PSE}} \neq \mathrm{ID}_{\mathrm{S}}$, but when the source is a sender then $\mathrm{ID}_{\mathrm{PSE}}=\mathrm{ID}_{\mathrm{PSO}} \neq \mathrm{ID}$, here $\mathrm{ID}_{\mathrm{S}}$ is the source's real ID and ID $\mathrm{PSO}_{\mathrm{PSO}}$ is the source's pseudo ID which we discussed in section 3.1 .

RRQSeqNO: Route request sequence number is used for identifying each route-request and corresponding route-reply packet from each other. It is generated by the source uniquely when source wants to communicate with a destination. $R R S e q N O=\mathrm{H}\left(\mathrm{ID}_{\mathrm{PSO}} \|\right.$ Time), where, $\mathrm{H}$ is a collision resistant hash function, $\mathrm{ID}_{\mathrm{PSO}}$ is a pseudo ID of the source, and Time is the calendar time when source generates $R R Q$ packet. This field remains unchanged for the corresponding $R R P$ generated by the destination.

ID $_{\mathrm{S}}$ : Source's ID ID , it is the source's real ID. Source generates a route request packet and puts its real ID in this field, and pseudo ID $\mathrm{ID}_{\mathrm{PSO}}$, in $\mathrm{ID}_{\mathrm{PSE}}$ field, thus $\mathrm{ID}_{\mathrm{PSE}}=\mathrm{ID}_{\mathrm{PSO}}$ but $\mathrm{ID}_{\mathrm{PSE}} \neq \mathrm{ID}_{\mathrm{S}}$. This is used by the destination to make a sign in route reply packet.

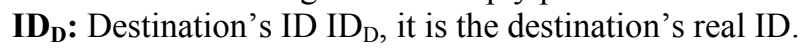

\section{RRP}

\begin{tabular}{|l|l|l|l|}
\hline ID $_{\text {PSE }}$ & ID $_{\text {PRE }}$ & RRQSeqNo & $\operatorname{Sign}_{\mathrm{D}}$ \\
\hline
\end{tabular}


ID $_{\text {PRE }}$ : Receiver's pseudo ID; on the path from the destination to the source when $R R P$ packet travels $\mathrm{ID}_{\mathrm{RPE}}$ defines the next node who receives $R R P$ packet.

Sign $_{\mathbf{D}}$ : Destination's Sign; when destination replies to source through intermediate nodes, it generates a sign, so that no one can forge. $\operatorname{Sign}_{\mathrm{D}}=\mathrm{H}_{2}\left(\mathrm{~K}_{\mathrm{DS}} \| R R Q S e q N O\right)$, where $\mathrm{K}_{\mathrm{DS}}$ is a session key between the source and the destination generated by the destination and defined as $\mathrm{K}_{\mathrm{DS}}=\mathrm{g}\left(\boldsymbol{\omega} \mathrm{H}_{1}\left(\mathrm{ID}_{\mathrm{D}}\right), \mathrm{H}_{1}\left(\mathrm{ID}_{\mathrm{S}}\right)\right)$ $=\mathrm{g}\left(\mathrm{H}_{1}\left(\mathrm{ID}_{\mathrm{D}}\right), \mathrm{H}_{1}\left(\mathrm{ID}_{\mathrm{S}}\right)\right)^{\boldsymbol{\omega}}$.

Destination also uses its session key $\mathrm{K}_{\mathrm{DS}}$, to decrypt data, sent by the source encrypted with source's session key $\mathrm{K}_{\mathrm{SD}}$, where $\mathrm{K}_{\mathrm{SD}}=\mathrm{g}\left(\boldsymbol{\omega} \mathrm{H}_{1}\left(\mathrm{ID}_{\mathrm{S}}\right), \mathrm{H}_{1}\left(\mathrm{ID}_{\mathrm{D}}\right)\right)=\mathrm{g}\left(\mathrm{H}_{1}\left(\mathrm{ID}_{\mathrm{S}}\right), \mathrm{H}_{1}\left(\mathrm{ID}_{\mathrm{D}}\right)\right)^{\boldsymbol{\omega}}$.

\subsection{Route Discovery and Route Reply}

On route discovery and route response phase the nodes maintain their corresponding table. When a node receives a $R R Q$ packet it broadcasts within its neighbor and when it receives a $R R P$ packet, it sends the $R R P$ corresponding to the receiver. RIOMO is described in terms of its functionalities which are described below.

\section{Route Discovery}

Every node in the network maintains its neighbor table with their pseudo IDs and corresponding session keys. When a source wants to communicate with a destination it generates a $R R Q$ and broadcasts this $R R Q$ within its neighbor to find a route, thus RIOMO is an on-demand routing protocol. By receiving a $R R Q$, a node checks $\mathrm{ID}_{\mathrm{D}}$ and $R R Q S e q N O$, of the $R R Q$ and makes the following decisions:

- If the node is the destination i.e., $I_{\mathrm{D}}$ matches with its real ID then it do the following tasks:

- It keeps $<R R Q S e q N O, \mathrm{ID}_{\mathrm{PSE}}>$ in its routing table; this $\operatorname{ID}_{\mathrm{PSE}}$ becomes $\operatorname{ID}_{\mathrm{PRE}}$ for $R R P$, generated by the destination. By replacing destination's own pseudo ID in the $\operatorname{ID}_{\mathrm{PSE}}$ field of $R R Q$, it broadcasts $R R Q$, within its neighbor. The purpose of this extra broadcast is to make attackers fool.

- It generates a $R R P$ with its own pseudo ID ID PSE$_{\text {, }}$, receiver's pseudo ID ID $_{\mathrm{PRE}}$ already discussed above, makes a sign $\operatorname{Sign}_{\mathrm{D}}$ discussed in section 4.2 and sends to the receiver. Notice that RRQSeqNO will be unchanged.

- If the node is not the destination and RRQSeqNO. is new, it keeps $R R Q S e q N O$, corresponding pseudo ID ID $\mathrm{IDE}_{\mathrm{PSE}}$ in its routing table, this information $<R R Q S e q N O$, $\mathrm{ID}_{\mathrm{PSE}}>$ is used by the node in the route reply procedure; this $\mathrm{ID}_{\mathrm{PSE}}$ becomes a receiver pseudo ID $\mathrm{ID}_{\mathrm{PRE}}$ in the route reply procedure. The node becomes a new sender and it puts its own pseudo ID in the $\mathrm{ID}_{\mathrm{PSE}}$ field of the $R R Q$ and this $R R Q$ within its region.

\section{Route Reply}

It is just a reverse path traverse of a $R R P$ explored by a $R R Q$. When a $R R Q$ reaches to the destination it generates a $R R P$ and forwards it in the reverse path as we discussed above. If a node receives a $R R P$, it checks $R R Q S e q N O$ in its routing table then updates receiver's pseudo ID ID $_{\mathrm{PRE}}$, with an appropriate $\operatorname{ID}_{\mathrm{PSE}}$ (i.e., from whom it receives the corresponding $R R Q$ with the same $R R Q S e q N O$ ), and sends in the reverse path. If source receives a $R R P$ it generates $\operatorname{Sign}_{\mathrm{S}}=\mathrm{H}_{2}\left(\mathrm{~K}_{\mathrm{SD}} \| R R Q S e q N O\right)$ and verify $\operatorname{Sign}_{\mathrm{D}}$. If $\operatorname{Sign}_{\mathrm{S}}=\operatorname{Sign}_{\mathrm{D}}$ the source sends data in the explored path by encrypting with its session key $\mathrm{K}_{\mathrm{SD}}$.

\subsection{Working Procedure in Brief}

1. Nodes make authentication of their neighbor nodes and maintain their neighbor table. Thus only the trusted nodes can take part in authentication.

2. On Route discovery phase, source generates a $R R Q$ and sends within its neighbor. If the destination is not within its neighbor then neighbor nodes become new sender. By replacing their own pseudo IDs broadcast within their own neighbor region. They also maintain this information in routing table as we discussed in section 4.3 . 
3. If the node is the destination it generates a $R R P$ and sends in the reverse path as we discussed in section 4.3

4. By receiving the $R R P$, source check the authenticity of the destination, if success then sends data in the explored path. Source and destination will use their corresponding session key for encryption and decryption as discussed in section 4.2 and 4.3 .

\section{Anonymity Achievement and Security Analysis}

When an $R R Q$ and $R R P$ travel from node to, every node generates a large bit random sequence corresponding to the fields of $R R Q$ and $R R P$. By extracting random bits from the fields of the packets, every node pads their own random bit sequence, and replaces their own pseudo IDs to the IDPSE $_{\text {PS }}$ accordingly. Thus the packets appear new when it moves from node to node. Also the fields (except ID $\mathrm{PSE}_{\text {, }}$ $\left.\mathrm{ID}_{\mathrm{PRE}}\right)$ are encrypted with corresponding session keys, thus it is also protected from intruders.

Identity Privacy: In RIOMO the identities of the nodes are represented by their pseudo IDs which are changed by the nodes in each session of communication. Pseudo IDs are also generated by using random numbers, hash functions as we discussed in section 3, also the control packets are encrypted so no one can recognize who is actual source and/or destination in a route request, route reply phase. Thus identity privacy of nodes is achieved in the network.

Location Privacy: If there is extra information added to control packets when the packets are forwarded form node to node; by observing the route request and the route response packets an attacker can estimation about the distance between the source and the destination. Thus, an attacker can set an attack regarding location privacy.

In our scheme, nodes do not know anything about the locations and identities of the other nodes in the network. So, no nodes in the network can determine the distance from them to the source and to the destination; they also do not know about the starting point of a packet traveling in the network. Only in a session the nodes know pseudo IDs of its neighbor region. Thus RIOMO ensures location privacy.

Route Anonymity: Current attacks on route anonymity are based on traffic analysis [24]. The general theory behind these kinds' of attacks is to trace or to find a path in which packets are moving. For these purpose the malicious nodes mainly looks for common information which are not changing in a packet during movements of control packets. As a result, the adversaries can find or to estimate the route from source to the destination. In RIOMO all the control packets appear new (Fig.2) to the network, when it travels form node to node. Because every time random bits are extracted and padded during movements of the control packets as we discussed at the beginning of this section. Thus route anonymity is achieved of a path.

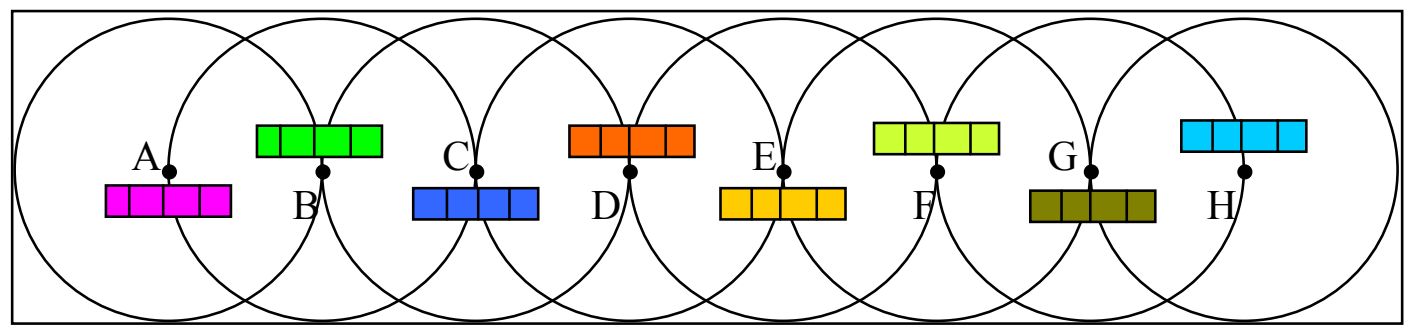

Fig. 2. Anonymity model; when packets move from node to node the packet fields are always appear new in the network.

DoS: According to the target of attack, multiple adversaries can co-operate or one adversary with enough power can target to a specific node to exhaust the resource of the node. For this purpose the adversaries try 
to identify a node and set a target to that specific node. In RIOMO identity privacy is achieved; so one can identify a node make a target to attack. Thus $D o S$ can be protected.

Wormhole Attacks: In wormhole attack an attacker records a packet in one location of the network and sends it to another location making a tunnel [25] between the attacker's nodes, later packet is retransmitted to the network under its control. Thus there could be a long distance travel for a packet to find a route from the source to the destination. In RIOMO an attacker can not be a trusted member within its neighbor so it can not be an intermediate node in route discovery or route reply phase thus an attacker can not take part in the routing. So the affect of the wormhole attack is not effective in AODPR.

Rushing Attack: By using the tunnel of wormhole attack an attacker can introduce rushing attack to rush packets. Existing on-demand routing protocol, such as AODV [7], DSR [8], LAR [26], Ariadne [27], SAODV [28], ARAN [29] and SRP [30], suffers from rushing attack. We discussed that RIOMO can prevent wormhole attack so rushing attack is not effective in this protocol.

\section{Conclusions and Future Works}

Anonymity is one of the important characteristics in securing a mobile ad-hoc network routing. In this paper an anonymous on-demand routing protocol, called RIOMO for preventing passive attacks, is proposed. In this protocol nodes take only one pseudo ID from system administrator and generate their own pseudo IDs for anonymous communications. Thus pseudo IDs maintenance cost is reduced compare to the existing protocol. Moreover RIOMO ensures node privacy, route anonymity and location privacy and is robust against several known attacks. Comparison analysis and security properties are described. As a further research we plan to make simulation with different criteria of performance analysis as well as implementation in a specific environment.

References

[1] P. Mohapatra and S. Krishnamurthy, "AD HOC NETWORKS: technologies and protocols", ISBN 0387-22689-3, Springer, pp. xxi-xxiii, 2005.

[2] Lou and Y. Fang, "A Survey on Wireless Security in Mobile Ad Hoc Networks: challenges and available solutions", Book chapter in Ad Hoc Wireless Networking, Kluwer, May 2003.

[3] Y. Guan, X. Fu, D. Xuan, P. Shenoy, R. Bettati, and W. Zhao, "NetCamo: Camouflaging Network Traffic for QoS-Guaranteed Mission Critical Applications", IEEE Transactions on Systems, Man, and Cybernetics, 31(4), pp.253-265, July 2001.

[4] DARPA. Research Challenges in High Confidence Networking. July 1998.

[5] O. Berg, T. Berg, S. Haavik, J. Hjelmstad, and R. Skaug, "Spread Spectrum in Mobile Communication", IEEE, 1998.

[6] S. Jiang, N. Vaidya, and W. Zhao, "Prevent Traffic Analysis in Packet Radio Networks", In Proceedings of DARPA Information Survivability Conference and Exposition (DISCEX II'01), Volume II- Volume 2, pp.1153-1158, June 2001.

[7] C. Perkins, E. Belding-Royer, and S. Das, "Ad hoc On-Demand Distance Vector (AODV) Routing," RFC 3561, July 2003.

[8] D. B. Johnson, D. A. Maltz, and Y. Hu, "The Dynamic Source Routing Protocol for Mobile Ad Hoc Networks (DSR)" < draft-ietf-manet-dsr-09.txt>, April 2003.

[9] B. Dahill, B. Levine, E. Royer, and C. Shields, "A Secure Routing Protocol for Ad Hoc Networks", University of Massachusetts Technical Report 01-37, 2001.

[10] ISO99 ISO IS 15408, 1999, available at http://www.commoncriteria.org/

[11] Yanchao Zhang, Wei Liu and Wenjing Lou, "Anonymous Communications in Mobile Ad Hoc Networks", In IEEE Infocom 2005, Miami, USA, March 13-17, 2005. The 24th Annual Conference Sponsored by IEEE Communications Society, available at http://ece.wpi.edu/ wjlou/publication/INFOCOM05_Zhang.pdf

[12] K. El-Khatib, L. Korba, R. Song, and G. Yee, "Secure dynamic distributed routing algorithm for ad hoc wireless networks", In International Conference on Parallel Processing Workshops (ICPPW'03), 2003. 
[13] M. G. Reed, P. F. Syverson, and D. M. Goldschlag, "Anonymous connections and onion routing", IEEE Journal on Selected Areas in Communications, Special Issue on Copyright and Privacy Protection, 16(4), pp. 482-494, 1998.

[14] J. Kong and X. Hong, "ANODR: ANonymous on demand routing with untraceable routes for mobile ad-hoc networks", In Fourth ACM International Symposium on Mobile Ad Hoc Networking and Computing (MobiHoc'03), pp. 291-302, 2003.

[15] Brian Neil Levine, Clay Shields, "Hordes: a multicast based protocol for anonymity", Journal of Computer Security Volume 10, Issue 3, ISSN: 0926-227X, pp. 213 - 240, 2002.

[16] A. Abdel-Hafez, A. Miri, and L. Orozco-Barbosa, "Authenticated Secure Communications in Wireless Networks".

[17] M. Ilyas. "The Handbook of Ad Hoc Wireless Networks". CRC Press, Washington D.C., 2003.

[18] D. Boneh, M. Franklin, "Identity Based Encryption from the Weil Pairing", SIAM Computing, Vol. 32, No. 3, pp. 586-615, 2003, Extended Abstract in Crypto 2001.

[19] P. S. L. M. Berreto, H. Y. Kim and M. Scott, "Efficient algorithms for pairing-based cryptosystems", Advances in Cryptology - Crypto '2002, LNCS 2442, pp.354-368, Springer-Verlag (2002).

[20] S. Galbraith, K. Harrison and D. Soldera, "Implementing the Tate Pairing", Algorithm Number Theory Symposium - ANTS V, LNCS 2369, pp. 324-337, Springer- Verlag (2002).

[21] D. Boneh and M. Franklin, "Identity-based encryption from the Weil pairing", Advances in Cryptology - CRYPTO’01, pp.213-229, Lecture Notes in Comput Sci. 2139 (2001).

[22] D. Boneh, B. Lynn and H. Shachum, "Short signatures from the Weil pairing", Advances in cryptology -ASIACRYPT'01, Lecture Notes in Comput Sci. 2248 (2001), 514-532.

[23] A. Joux and K. nguyen, "Separating decision Diffie-Hellman from Diffie-Hellman in Cryptographic groups", Cryptology ePrint Archive, Report 2001/03, available at http://eprint.iacr.org/2001/03/.

[24] Raymond, J.-F., "Traffic Analysis: Protocols, Attacks, Design Issues and Open Problems," in Proceedings of PET 01, Vol. 2009, LNCS, pp. 10-29, Springer-Verlag, 2001.

[25] Y.C. Hu, A. Perrig, and D. B. Johnson, "Packet leashes: A defense against wormhole attacks in wireless ad hoc networks", In Proceedings of the Twenty-Second Annual Joint Conference of the IEEE Computer and Communications Societies (INFOCOM 2003), 2003.

[26] Young-Bae Ko and Nitin Vaidya, "Location-Aided Routing (LAR) in Mobile Ad Hoc Networks", In Proceedings of the Fourth International Conference on Mobile Computing and Networking (MobiCom'98), pp. 66-75, October 1998.

[27] Yih-Chun Hu, Adrian Perrig, and David B. Johnson, "Ariadne: A Secure On-Demand Routing Protocol for Ad Hoc Networks", In Proceedings of the Eighth Annual International Conference on Mobile Computing and Networking (MobiCom 2002), pp. 12-23, September 2002.

[28] Manel Guerrero Zapata and N. Asokan, "Securing Ad Hoc Routing Protocols," In Proceedings of the ACM Workshop on Wireless Security (WiSe 2002), September 2002.

[29] Kimaya Sanzgiri, Bridget Dahill, Brian Neil Levine, Clay Shields, and Elizabeth Belding-Royer, "A Secure Routing Protocol for Ad hoc Networks", In Proceedings of the 10th IEEE International Conference on Network Protocols (ICNP '02), November 2002.

[30] Panagiotis Papadimitratos and Zygmunt J. Haas, "Secure Routing for Mobile Ad Hoc Networks", In SCS Communication Networks and Distributed Systems Modeling and Simulation Conference (CNDS 2002), January 2002. 